\title{
A New Interpolation Algorithm in Fast Radiative Transfer Model
}

\author{
Yu Yi ${ }^{1,2}$, Zhang Weimin ${ }^{1}$, Huang Qunbo ${ }^{1}$, Yao Lu ${ }^{1}$ \\ 1. Computer Science, University of National Defense Technology, Changsha, China \\ 2. NO.94865 Troops of Chinese People's Liberation Army, Hangzhou, China \\ yuyi2019@nudt.edu.cn wmzhang104@163.com hqb09@163.com shaoq@163.com
}

\begin{abstract}
Many schemes of interpolation can be used for mapping from various coordinates of the numerical weather predictions (NWP) to the vertical coordinates of data assimilation systems. While most of the schemes do not imply the availability of a corresponding vertical mapping between the coordinates, even they are not implemented expediently. This leads to very noisy gradient with large unrealistic spikes which are simulated by the fast radiance transfer(RT) model for the first guest values from the numerical weather system and, consequently to incorrect data assimilation. The memorandum analyses a new algorithm of piecewise weighted integral interpolation applied in the Radiative Transfer for TIROS Operation Vertical Sounders (RTTOV). Experimental results show that the internal interpolation incorporated into RTTOV can map the coordinate of NWP model to the coordinate of data assimilation system flexibility, and can use the atmospheric information of the profiles on coordinate of NWP model sufficiently. Also the internal RTTOV interpolation can avoid "blind" levels derived from the nearest-neighbor log-linear interpolator and successfully retain the smooth structure of the radiance gradients.
\end{abstract}

Keywords-Data Assimilation; NWP Model; RTTOV; Interpolation

\section{INTRODUCTION}

The data assimilation of satellite radiances[1] for nadir sounders involves fast radiance transfer (RT) models which simulates atmospheric profiles of temperature, humidity, ozone and etc. predicted as the first guess (FG) values by the numerical weather prediction model (NWP) to be the radiance from the corresponding observation location or the radiance gradient value used in operation. The atmospheric profiles distribute in the vertical pressure coordinates of the NWP models. The NWP model has different vertical coordinates [2], typically terrain-following such as $\sigma$ or $\eta$, where $\sigma \mathrm{i}=\mathrm{Ps} / \mathrm{Pi}, \eta \mathrm{i}=(\mathrm{Pi}-\mathrm{Ptop}) /(\mathrm{Ps}-\mathrm{Ptop})$, with $\mathrm{Pi}$ being the pressure at level i, Ptop the pressure at the model top and Ps the surface pressure. Also the RT model has various vertical coordinates, such as the Radiative Transfer for TIROS Operational Vertical Sounder (RTTOV; Matricardi et al.,2004), are evaluated on fixed pressure levels to compute transmittance while, the Optical Path Transmittance (OPTRAN; McMillin et al.,2005) use different levels. The mismatch of coordinate systems in NWP models and RT models makes it necessary to introduce an interpolation algorithm that maps the NWP model coordinate to the RT model coordinate for the various data assimilation systems.

There are many algorithms of vertical interpolation, such as linear, piecewise linear, or spine. Facts have proved that most interpolation algorithms do not imply the availability of a corresponding coordinate mapping in operations. In additional, various meteorological forecast centers use the interpolator between the coordinates in NWP models and RT models alternatively in different ways. For example, the Canadian Middle Atmosphere Model (CAMA; Beagley et al., 1997; Polavarapu et al., 2005) employed the nearestneighbor log-linear interpolator in the NWP model [2]. RT model computes the radiative transfer calculation for the atmospheric profiles interpolated by the NWP model. The effect of this was that model-level became "blind" levels in areas where the RT vertical resolution is much poorer than that of NWP (e.g., upper stratosphere), as these model levels contributed little or not at all to the radiance calculations [3]. RT model could not use the information sufficiently from the atmospheric profiles which are the FG values derived from the NWP model. This leads to incorrect mapped Jacobians which may result in less optimal or incorrect increment solutions, thus implying potentially poorer quality analyses and model forecasts in next step. This memorandum analyses and investigates a new algorithm of piecewise weighted integral interpolation applied in the Radiative Transfer for TIROS Operation Vertical Sounders (RTTOV) used by several operational weather forecasting centers. The internal RTTOV interpolation can use the information of atmospheric profiles relying on the coordinates of the NWP model efficiently and flexibly to simulate the radiance or radiance Jacobians required by the data assimilation calculations.

\section{DATA ASSIMILATION AND INTERPOLATION}

The basic idea of the four-dimensional (4D) variational assimilation [4] for atmospheric data is to optimally combine observations yi over short time window and the corresponding model short-term forecast $\mathrm{xb}$ on the model grid. In its general form, it is defined as the minimization of the following cost function:

$$
J(x)=\frac{1}{2}\left(\mathrm{x}^{-\mathrm{x}_{\mathrm{b}}}\right)^{\mathrm{T}} \mathrm{B}^{-1}\left(\mathrm{x}-\mathrm{x}_{\mathrm{b}}\right)+\sum_{i=0}^{n}\left(\mathrm{y}_{i}-H_{i}\left[\mathrm{x}_{i}\right]\right)^{\mathrm{T}} \mathrm{R}_{i}^{-1}\left(\mathrm{y}_{i}-H_{i}[\mathrm{x}]\right)
$$

This equation provides the observation operators Hi(xi) which are generalized to include a forecast model that will allow a comparison between the model state and observations at the appropriate time. By defining $\delta x=x-x b$, the tangent linear approximation for $\mathrm{H}(\mathrm{x})$ can be used: $\mathrm{H}(\mathrm{x}) \approx \mathrm{H}(\mathrm{xb})+\mathrm{H} \delta \mathrm{x}$, where $\mathrm{H}$ is the Jacobian matrix $\mathrm{dH}(\mathrm{x}) / \mathrm{d}(\mathrm{x})$. Its adjoint HT (superscript T denotes transpose) is used by a descent algorithm which provides a solution $\mathrm{x}$. we denote by the subscript $i$ the quantities at any given observation time $i$. Hence, yi and xi are the observations and the model at time i, and $\mathrm{Ri}$ is the error covariance matrix for the observation 
errors. The background error covariance matrix B is only defined at initial time, the time of the background $\mathrm{xb}$ and of the analysis xa. Using unprimed and primed variables for the NWP and RT model coordinates respectively, we can linearly interpolate the atmospheric profile $\mathrm{x}^{\prime}$ on the RT model coordinate from NWP coordinate by:

$$
x^{\prime}\left(p_{i}\right)=x_{i}^{\prime}=s_{i}(\mathrm{x})=\sum_{j} W_{i, j} x_{j} \text { or } \mathrm{x}^{\prime}=\mathrm{Wx}
$$

The interpolator si leads to the interpolator matrix $\mathrm{W}$. The weights Wi,j are a function of distance in $\ln (\mathrm{p})$ and sum over $\mathrm{j}$ to unity. Therefore we can write the RT model application associated with individual radiance observations as $\mathrm{H}\left(\mathrm{x}^{\prime} \mathrm{b}\right)=\mathrm{H}(\mathrm{Wxb})$. Now, mapping the Jacobians $\partial \mathrm{f} / \partial x^{\prime}$ requires that

$$
h_{j}=\left.\frac{\partial f}{\partial x_{j}}\right|_{x}=\left.\sum_{i} \frac{\partial f}{\partial x_{i}^{\prime}}\right|_{x} \frac{\partial x_{i}^{\prime}}{\partial x_{j}}=\left.\sum_{i} \frac{\partial f}{\partial x_{i}^{\prime}}\right|_{x} W_{i, j} \quad \text { or } \mathrm{h}=\mathrm{W}^{\mathrm{T}} \mathrm{h}
$$

The gradient $\partial x_{i}^{\prime} / \partial x_{j}$ leads to the transpose of the interpolator matrix, the adjoint matrix WT. The tangent linear matrix operator $\mathrm{H}$ consisted of all kinds of onedimensional (1D) arrays hT, accordingly, HT=WTH'T. If the interpolator matrix $\mathrm{W}$ does not involve some of the NWP levels within the non-zero range of Jacobians in $\mathrm{h}^{\prime}$, then the interpolator adjoint matrix WT will incorrectly assign null values to the corresponding Jacobians in h. From a case of a single observation $(\mathrm{H}=\mathrm{hT})$ we can easily see this effect and a near-linear inverse problem, i.e. $H(x)=H(x b)+H \delta x$, so the solution to the minimization of the cost function is

$$
\mathrm{x}_{\mathrm{a}}=\mathrm{x}_{\mathrm{b}}+\mathrm{BH}^{\mathrm{T}}\left(\mathrm{HBH}^{\mathrm{T}}+\mathrm{R}\right)^{-1}\left(\mathrm{y}-H\left(\mathrm{x}_{\mathrm{b}}\right)\right)
$$

Obviously, null values in hT imply no changes in the corresponding element of $\mathrm{x}$ in the case of a diagonal covariance B.

The interpolator can impact on the observation operators $\mathrm{Hi}(\mathrm{xi})$ in the minimization calculation of the cost function and its tangent linear approximation operator $\mathrm{H}$ in addition to the observations and the forecast $\mathrm{xb}$, and therefore can influence the quality of the solution $x a$ and the solution increments $(\mathrm{xa}-\mathrm{xb})$. This, in turn, impacts the quality of the following forecast from the NWP model and the forecast's differences from the observations [3].

\section{Algorithm Based On Piecwise Weighted INTEGRAL INTERPOLATION}

A necessary condition for mapping effectively the vertical coordinates between the NWP model and RT model is that all the NWP levels [2] over the non-zero region of the atmospheric profiles as the NWP first guess values must be involved in the interpolator. Moreover, the resultant level of the participation of the NWP coordinate with equal space distance to the interpolator should be equal with weight of unity. A new interpolator can satisfy these conditions based on the piecewise weighted integral interpolation algorithm. It can be constructed from the sample averaging integral over a pair of successive destination coordinate layers, e.g. RT model coordinate layers, and this method can make sure participation of all relevant points from the source coordinate, e.g. the NWP coordinate. Use the $\ln (p)$ to present $z$, the weighted averaging integral[2] over the range zi-1 to zi+1 for the interpolation from the NWP level to an RT model level $\mathrm{i}$ is

$$
x_{i}^{\prime}=\frac{\int_{i}^{i+1} w_{i}(z) x(z) d z+\int_{i-1}^{i} w_{i}(z) x(z) d z}{\int_{i}^{i+1} w_{i}(z) d z+\int_{i-1}^{i} w_{i}(z) d z}
$$

Where, for piecewise weighting,

$$
w_{i}(z)=\left\{\begin{array}{c}
1-\left(\frac{z-z_{i}^{\prime}}{z_{i-1}^{\prime}-z_{i}^{\prime}}\right), \quad \text { for } z_{i-1}^{\prime}<z<z_{i}^{\prime} \\
1-\left(\frac{z-z_{i}^{\prime}}{z_{i+1}^{\prime}-z_{i}^{\prime}}\right), \quad \text { for } z_{i}^{\prime}<z<z_{i+1}^{\prime} \\
0 \quad \text { for } z \leq z_{i-1}^{\prime}, z \geq z_{i+1}^{\prime}
\end{array}\right.
$$

It can be easily seen that the pressure increase with increasing index value and with weights of unity at $\mathrm{zi}^{\prime}$ and zero at $\mathrm{zi} \pm 1^{\prime}$. In addition, in order to ensure that all NWP levels in the overlapping regions are involved, a linear weighting function in $\mathrm{z}$ can also ensure every NWP coordinate with consistency, when both spaces between the two coordinates and the target level are equal.

An interpolation algorithm based on nearest-neighbor log-linear interpolator is commonly applied in data assimilation,

$$
x\left(z_{i}^{\prime}\right)=\frac{\left(z_{j+1}-z_{i}^{\prime}\right) x_{j}+\left(z_{i}^{\prime}-z_{j}\right) x_{j+1}}{\left(z_{j+1}-z_{j}\right)} \text {,where } z_{j+1} \leq z_{i}^{\prime} \leq z_{j}
$$

Figure 1 shows the weighting function for two different distributions of NWP levels. The piecewise weighted integral interpolator of Equation (5), referred to as Interpolator 1, while commonly used nearest-neighbor loglinear interpolator of Equation (7), referred to as Interpolator 2.

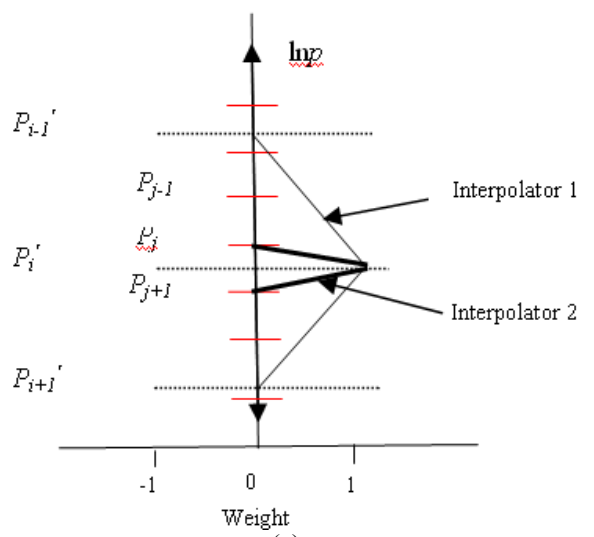

(a) 


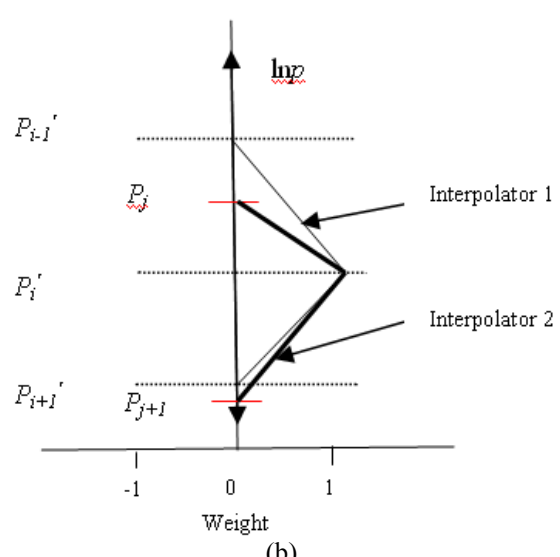

(b)

Figure 1. Weighting function interpolator 1 (thin lines) and interpolator 2 (thick lines).

(a) and (b) show weighting function for two different distributions of NWP levels.

\section{IMPLEMETATION AND ANALYSis OF THE NEW INTERPOLATOR IN RTTOV}

\section{A. Implementation of the interpolation algorithm}

RTTOV calculates the top of the atmosphere radiance or equivalent brightness temperature $\mathrm{TB}$ in channel of a given sensor for the input atmospheric profile in each run. Two sets of interpolation [5] are involved in the calculation, one for profile of variables $\mathrm{X}$, and the other for the accumulated optical depths $\mathrm{D}$, both using the piecewise weighted integral interpolation algorithm described in section 3 .

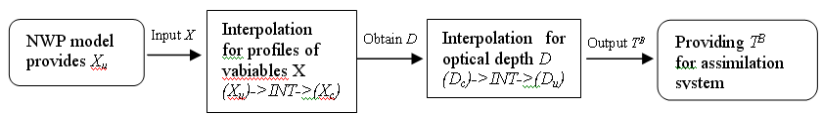

Figure 2. Two sets of interpolation in RTTOV

In Figure 2, the parentheses imply matrix, and the subscript $\mathrm{u}$ and $\mathrm{c}$ mean respectively the user levels and the RTTOV levels defined in the coefficient files. In order to implement the internal interpolation, RTTOV introduces the concept of "user levels" [5] when reading the input profiles and calculating the radiance or radiative gradients for these profiles. The user levels are specified by the user and can be understood as the NWP model pressure levels. In older versions, the user was required to provide profiles already interpolated to the fixed pressure levels. The effect of this was that model-level became "blind" levels in areas where the RTTOV vertical resolution is much poorer than that of NWP (e.g., upper stratosphere), as these model levels contributed little or not at all to the radiance calculations. With the interpolator implemented in RTTOV, the computational process is also made relevant adjust [6], allowing the input profiles derived from the NWP model on different user levels. This means the pressure values may differ from one profile to the next in any run, but users need to ensure that the profile cover the whole atmosphere covered with an adequate number of levels at least close to the coefficient levels or more. A coarse layering will reduce the accuracy of the calculations.

Table 1 shows the parameters of the piecewise weighted integral interpolation algorithm.

TABLE I. INPUT PARAMETERS AND OUTPUT PARAMETERS OF THE PIECEWISE WEIGHTED INTEGRAL INTERPOLATION ALGORITHM

\begin{tabular}{|c|c|}
\hline Input parameter & meaning \\
\hline PX1 (KN1) & Levels of output domain \\
\hline PX2(KN2) & Levels of input domain \\
\hline KN1 & Dimension of PX1 \\
\hline KN2 & Dimension of other arrays \\
\hline $\begin{array}{c}\text { Output } \\
\text { parameter }\end{array}$ & $\begin{array}{c}\text { meanig } \\
\text { interpolation from input to output domains }\end{array}$ \\
\hline PZ(KN,KN1) & Start index for relevant PZ row array segment \\
\hline Kstart (KN1) & End index for relevant PZ row array segment \\
\hline Kend (KN1) & Returns error status to allow a soft failure \\
\hline Errorstatus &
\end{tabular}

The piecewise weighted integral interpolation implemented in the RTTOV increases the weights of contribution from the lowest and highest input domain levels for output layers intersecting these input domain boundaries. It consists of applying constant value extrapolation by introducing 'fake' layers. For the surface of the input domain, as example, this implies creating a virtual surface layer which extends to the lower boundary of the output domain layer which contains the input domain surface. This increases the contributing weight of the surface which would be otherwise underestimated in the original code due to the interpolator actually doing piecewise weighted averaging.

The algorithm process as follows:

Assumptions: PX1(i) $<$ PX1(i+1) \& PX2(i) $<$ PX2(i+1)

Set integration range boundaries for output domain (z1,z3)

Increase the contribution of layers from the boundaries

If $(\mathrm{px} 2(\mathrm{j}+1)>\mathrm{z} 1)$ then

Add the weight contribution of layers in (ki-1 to ki)

If $(\mathrm{px} 2(\mathrm{j}+1) \geq \mathrm{z} 2)$ then

Add the weight contribution of layers in (ki to ki+1)

Loop through accumulating weight contributions

Normalize sum of weights to unity

B. Experimental results
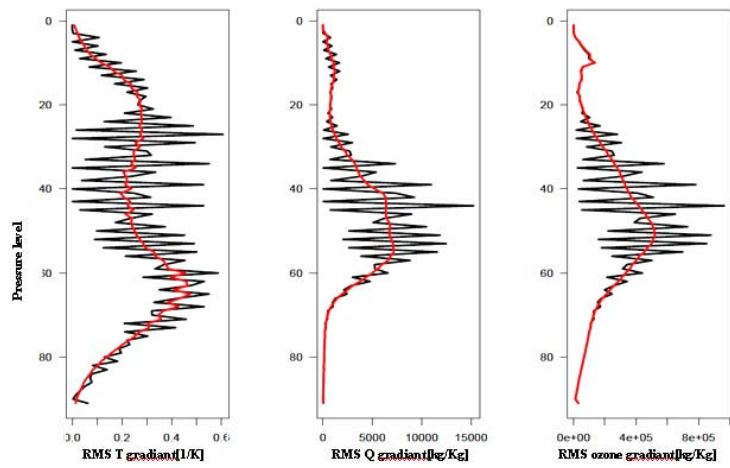

Figure 3. initial temperature, humidity, and the ozone gradients 
The impact of the interpolation has been evaluated with extended experiments. The RTTOV experiment uses the piecewise weighted integral interpolation incorporated in RTTOV model, whereas the NWP experiment uses the nearest- neighbor log-linear interpolation applied in the NWP model. The profiles after interpolation are used for the radiative simulation calculation [1], and then the data assimilation system use the radiance compared to the observations to generate the root mean square (RMS) of the initial temperature, humidity, and the ozone gradients (Figure 3).

The RTTOV internal interpolation improves the gradients from the radiative transfer simulation, calculated for the minimization of the data assimilation cost function. While the nearest-neighbor log-linear interpolation in NWP leads to very noisy gradients with large unrealistic spikes, the internal RTTOV interpolation successfully retains the smooth structure of these gradients. This is a noticeable improvement, and is a result of the smoother nature of the piecewise weighted integral interpolation.

\section{CONCLUTION}

This memorandum analyses the importance of the interpolation algorithm to the data assimilation, and introduce the new piecewise weighted integral interpolation algorithm incorporated in the RTTOV model. This interpolation can map flexibly the coordinate of the NWP model to the coordinate of the data assimilation system [7], and can use the atmospheric information sufficiently to avoid the spikes and "blind" [3] levels in the gradients otherwise commonly encountered when using the nearest-neighbor loglinear interpolation. The move to the RTTOV interpolation is not merely a replacement of one interpolation method with another. Three aspects contribute:

- using a smoother interpolator which leads to a different atmospheric expression for the optical depth integration,
- the use of the fully NWP temperature profile to specify the source function in the radiative transfer integration, and

- the reduced influence of RTTOV's unrealistic isothermal layer assumption near the top of the atmosphere, thus the simulation of the contribution from the top of the atmosphere is more realistic.

\section{ACKNOWLEDGMENT}

This research was supported by the National Natural Science Foundation of China under Grant No.40775064.

Figure 3 Root mean square of the initial temperature (left), humidity (middle), and ozone (right) gradients for all assimilated

radiance observations. Results with the NWP interpolation are show in black, with the RTTOV internal interpolation in red.

\section{REFERENCES}

[1] Roger Saunders; RTTOV-9 User Guide; http://research.metoffice. gov.uk/research/intproj/nwpsaf/rtm/rttov9_files/rttov9_svr.pdf;2010.

[2] Y. J. Rochon, * L. Garand, D. S. Turner, and S. Polavarapu; Jacobian mapping between vertical coordinate systems in data assimilation; QUARTERLY JOURNAL OF THE ROYAL METEOROLOGICAL SOCIETY;2007

[3] Niels Bormann, D. Salmond, MarcoMatricardi, A. Geer, and M. Hamrud; The RTTOV-9 upgrade for clear-skyradiance assimilation in the IFS;ECMWF Technical Memoranda;2009.

[4] Eugenia Kalnay; Atmospheric Modeling, Data Assimilation and Predictability; Press Syndicate of the University of Cambridge;2003.

[5] Roger Saunders; RTTOV-9 science and validation report;http://research.metoffice.gov.uk/research/interproj/nwpsaf/rtm/ rttov9_files/rttov9_svr.pdf;2010-10-13.

[6] Roger Saunders; RTTOV v10 top level design; http://research.metoffice.gov.uk/research/interproj/nwpsaf/rtm/docs_r ttov_v9_tld.pdf;2011.

[7] Matricardi; RTIASI-4, a new version of the ECMWF fast radiative transfer model for the infrared atmospheric sounding interferometer. Technical Memorandum ; 2003. 